\title{
Temperley-Lieb Words as Valence-Bond Ground States
}

\author{
Peter F. Arndt†, Thomas Heinzel† and C.M. Yung $\ddagger$ \\ $\dagger$ Physikalisches Institut \\ Universität Bonn, Nussallee 12, 53115 Bonn, Germany \\ $\ddagger$ Department of Mathematics \\ Australian National University,Canberra, ACT 0200, Australia
}

\begin{abstract}
Based on the Temperley-Lieb algebra we define a class of one-dimensional Hamiltonians with nearest and next-nearest neighbour interactions. Using the regular representation we give ground states of this model as words of the algebra. Two point correlation functions can be computed employing the Temperley-Lieb relations. Choosing a spin- $\frac{1}{2}$ representation of the algebra we obtain a generalization of the ( $q$-deformed) MajumdarGhosh model. The ground states become valence-bond states.
\end{abstract}

BONN HE-94-26

cond-mat/9411085

November 1994 
In this paper we present a class of one-dimensional Hamiltonians $H=H(a, b, q)$ with nearest and next-nearest neighbour interaction. The Hamiltonian is given in terms of elements of a Temperley-Lieb algebra [1] and has the structure of the MajumdarGhosh model [2, 3, 4, 5, 6]. With specific representations of this algebra one obtains various quantum spin chains. The functions $a=a(q)$ and $b=b(q)$ determine the next-nearest neighbour interaction and can be chosen such that the ground state can be given explicitly. In a graphical form of the regular representation of the Temperley-Lieb algebra [7, 8] these ground states have a particular simple form. They are related to valence-bond spin states. We also calculate correlation functions using the graphical representation.

A Temperley-Lieb algebra $T_{N}(q)$ is defined by the following relations on the generators $e_{i}, i=1,2, \ldots, N-1$ :

$$
\begin{aligned}
& e_{i} e_{i}=x e_{i}=\left(q+q^{-1}\right) e_{i} \\
& e_{i} e_{i \pm 1} e_{i}=e_{i} \\
& e_{i} e_{j}=e_{j} e_{i} \quad(j \neq i \pm 1)
\end{aligned}
$$

Here we consider the case where $q$ is real. These algebras appear as centralizer algebras of the quantum group $U_{q} S U(2)[0,9]$. Therefore $U_{q} S U(2)$ invariant models naturally show an underlying Temperley-Lieb structure. However, our results can be used for models having other quantum group symmetries as well [10, 11, 12, 13.

We define the Hamilton operator as an abstract element of the TemperleyLieb algebra. We use the two-point correlation operators defined in [8]. These operators preserve the quantum group symmetry of the respective representations of the Temperley-Lieb algebra. Two types of two-point operators can be defined by the recursive relations

$$
\begin{aligned}
& g_{l, l+1}=g_{l, l+1}^{ \pm}=e_{l}-\left(q+q^{-1}\right)^{-1} \quad 1 \leq l \leq N-1 \\
& g_{l, m}^{ \pm}=-q^{ \pm 1} g_{l, n}^{ \pm} g_{n, m}^{ \pm}-q^{\mp 1} g_{n, m}^{ \pm} g_{l, n}^{ \pm} \quad 1 \leq l<n<m \leq N \\
& g_{m, l}^{ \pm}=q^{\mp 4} g_{l, m}^{ \pm} \quad 1 \leq l<m \leq N .
\end{aligned}
$$

In the following we use only $g_{l, m}^{ \pm}$with $l<m$. Note that the definition is in terms of generators $e_{l}$ of $T_{N}(q)$, independent of their realization.

We employ both $g_{l, m}^{+}$and $g_{l, m}^{-}$to define the Hamilton operator

$$
H^{(N)}(a, b, q)=\frac{2-x^{2}}{2 x\left(x^{2}-1\right)} \sum_{i=1}^{N-2} h_{i, i+1, i+2}+2 a(q)(N-2)\left(2-x^{2}\right)^{2}
$$

with

$$
\begin{aligned}
h_{i, i+1, i+2}= & 2 g_{i, i+1}+2\left(\left(x^{2}-2\right) a(q)-1\right) g_{i+1, i+2} \\
& +a(q)\left(g_{i, i+2}^{+}+g_{i, i+2}^{-}\right)+b(q)\left(g_{i, i+2}^{+}-g_{i, i+2}^{-}\right) .
\end{aligned}
$$


The operator $h_{i, i+1, i+2}$ acts on sites $i, i+1, i+2$ and involves the generators $e_{i}$ and $e_{i+1}$. The value of $x$ is given by the Temperley-Lieb relation (1a). The arbitrary functions $a=a(q)$ and $b=b(q)$ are weighting factors of the symmetric and antisymmetric contribution of $g_{i, i+2}^{+}$and $g_{i, i+2}^{-}$to the Hamiltonian. For $q=1$ the Hamiltonian is independent of $b$, since $g_{l, m}^{+}=g_{l, m}^{-}$. It is useful to notice how the Hamiltonian changes, if we replace $q$ by $1 / q$. We have the identity

$$
H^{(N)}(a, b, q)=H^{(N)}(a,-b, 1 / q) .
$$

The coefficients in (4) are chosen such that simple ground states can be found. To this end we use the regular representation of $T_{N}(q)$ on boundary diagrams [7]. We find the ground states to be specific Temperley-Lieb words. A word in this context means a straight product of generators and is realized by a single boundary diagram.

A boundary diagram is given by two rows of $N$ upper and $N$ lower points with $N$ non-intersecting lines connecting the points such that any point is connected to a single other point. A generator is realized as

$$
e_{i}=||_{2} \ldots\left|\bigodot_{i=1}^{\cup}\right| \cdots||_{N-1 N} .
$$

The composition of two words is defined as stacking the corresponding diagrams on top of each other and identifying the lower points of the first with the upper points of the second diagram. Any closed line appearing in this process is discarded from the diagram and replaced by a factor $x=q+q^{-1}$. This reflects the relation $(1 a)$. The other Temperley-Lieb relations $(1 b)$ and $(1 c)$ can easily be verified by drawing the corresponding diagrams. By successive composition of generators (6) all distinct words of $T_{N}(q)$ are realized as different boundary diagrams. Thus for calculations in the regular representation of $T_{N}(q)$ we can take all possible boundary diagrams as a basis of the representation space.

In the following we give eigenvectors and eigenvalues of $H^{(N)}$. First we take $N$ even. Employing boundary diagrams it is straightforward to show that (3) has the eigenvector

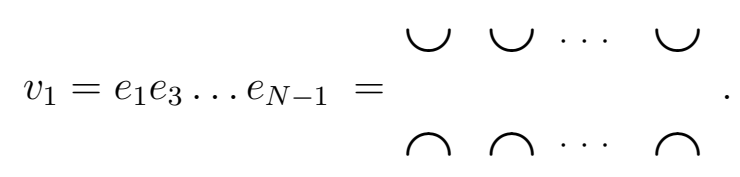

The additive constant in (3) is chosen such that its eigenvalue is zero.

If we impose a condition on the functions $a(q)$ and $b(q)$ :

$$
a=\left(2-x\left(q-q^{-1}\right) b\right) /\left(x^{2}-1\right),
$$


we find two further eigenwords with eigenvalue zero:

$$
v_{2}=\left(e_{2} e_{4} \ldots e_{N-2}\right) v_{1}=\underbrace{\bigcup \cdots \cap}_{\frown \cap \cup \cdots \cup}
$$

and

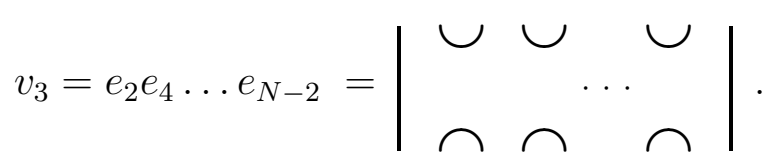

For an odd number of sites $N$ and the condition (8) we have two eigenwords that are given by the diagram (10) without the left or right vertical line. We denote these words as $v_{4}=e_{1} e_{3} \ldots e_{N-2}$ respectively $v_{5}=e_{2} e_{4} \ldots e_{N-1}$. Their eigenvalues can be computed as $\mp b / 2\left(q^{2}+q^{-2}\right)\left(q-q^{-1}\right)\left(q+q^{-1}\right)^{-1}$ for $v_{4}$ respectively $v_{5}$.

For the following we impose condition (8). We investigate whether we can choose the function $b(q)$ such that the eigenstates $v_{i}$ given above become ground states of the Hamiltonian $H^{(N)}$. We can show that $H^{(N)}$ has a ground state $v_{i}$, if the function $b(q)$ is bounded by

$$
b \leq(\geq) \frac{q^{3}+2 q+2 q^{-1}+q^{-3}}{q^{5}+2 q^{3}-2 q^{-3}-q^{-5}} \quad \text { for }|q|>1(|q|<1)
$$

The relation between the cases $|q|>1$ and $|q|<1$ reflects the symmetry (5).

For a proof first consider the case $N$ even. One can think of $H^{(N)}$ as the sum of $N / 2-1$ Hamilton operators $H^{(4)}$ each involving only three generators. This can be seen by grouping together $h_{i, i+1, i+2}$ and $h_{i+1, i+2, i+3}(i$ odd) in the definition (3)). The operator $H^{(4)}$ can be diagonalized (see below). Hence the lowest eigenvalue of $H^{(N)}$ is bounded from below by $N / 2-1$ times the lowest eigenvalue of $H^{(4)}$. We take the 14 possible boundary diagrams with $N=4$ as a basis to write $H^{(4)}$ in the regular representation. Diagonalization of this $14 \times 14$ matrix gives the eigenvalues

$$
\begin{aligned}
\lambda_{1}= & 0 \\
\lambda_{2}= & 2-\left(q-q^{-1}\right) b(q) \\
\lambda_{3}= & 1-\left(q-q^{-1}\right)\left(q+q^{-1}\right)^{-1} b(q) \\
\lambda_{4}= & 1-\left(q^{3}-q^{-3}\right)\left(q+q^{-1}\right)^{-1} b(q) \\
\lambda_{5}= & 1-\left(q^{5}+2 q^{3}-2 q^{-3}-q^{-5}\right)\left(q^{3}+2 q+2 q^{-1}+q^{-3}\right)^{-1} b(q) \\
\lambda_{6 / 7}= & 1-\frac{1}{2}\left(q^{3}+2 q+2 q^{-1}+q^{-3}\right)^{-1}\left[\left(q^{5}+2 q^{3}+q-q^{-1}-2 q^{-3}-q^{-5}\right) b(q)\right. \\
& \pm\left[\left(q^{10}+6 q^{6}-2 q^{4}+q^{2}-12+q^{-2}-2 q^{-4}+6 q^{-6}+q^{-10}\right) b^{2}(q)\right. \\
& \left.\left.-8\left(q^{4}+q^{2}-q^{-2}-q^{-4}\right) b(q)+4\left(q+q^{-1}\right)^{2}\right]^{\frac{1}{2}}\right] .
\end{aligned}
$$


The degeneracy of the first eigenvalue is seven and of the second two. The others are non-degenerate. From this result we can conclude that, if we have equation (11), all eigenvalues of $H^{(4)}$ are greater or equal to zero. Hence for functions $b(q)$, that fulfill conditions (8) and (11), $v_{1}, v_{2}$ and $v_{3}$ are ground states of the Hamiltonian $H^{(N)}$ because their eigenvalue is zero.

For $N$ odd we can analogously view $H^{(N)}$ as the sum of $(N-3) / 2$ operators $H^{(4)}$ and one operator $H^{(3)}$. Making use of the 5 different boundary diagrams for $N=3$ we find the eigenvalues of $H^{(3)}$ :

$$
\begin{aligned}
& \mu_{1}=1-\frac{b(q)}{2}\left(q^{2}-q^{-2}\right) \\
& \mu_{2 / 3}= \pm \frac{b(q)}{2}\left(q^{2}+q^{-2}\right)\left(q-q^{-1}\right)\left(q+q^{-1}\right)^{-1} .
\end{aligned}
$$

The eigenvalues $\mu_{2}$ and $\mu_{3}$ are twofold degenerate, $\mu_{1}$ is non-degenerate. If we impose condition (11), $\mu_{1}$ is always positive. Note that for (8) the value $\mu_{2}\left(\mu_{3}\right)$ is also an eigenvalue of $H^{(N)}$ with eigenvector $v_{4}\left(v_{5}\right)$. Thus imposing both conditions (8) and (11) a ground state of $H^{(N)}$ is given by $v_{4}$ or $v_{5}$ depending on the values of $q$ and $b$. Explicitely, for $|q| \geq 1$ and $b \leq 0(b \geq 0)$ we have $v_{4}\left(v_{5}\right)$ as ground state of $H^{(N)}$. For $|q| \leq 1$ and $b \leq 0(b \geq 0)$ we find the ground state $v_{5}\left(v_{4}\right)$.

If we drop condition (8), $v_{1}$ remains eigenstate of $H^{(N)}$ for $N$ even. For this case we want to remark, that one can choose two functions $a(q)$ and $b(q)$ within certain bounds to make $v_{1}$ a ground state of the Hamiltonian.

Next we calculate correlation functions for the ground states $v_{1}$ and $v_{2}$. Again the computation is completely general in terms of boundary diagrams.

For a graphical calculation of the correlation functions we have to restrict our attention to the words of the left-sided ideal that is generated by $v_{1}=e_{1} e_{3} \ldots e_{N-1}$ $\left(N\right.$ even). The diagrams with lower part as in $v_{1}$ and arbitrary upper part constitute this ideal $\mathcal{S}$. Thus the ground states $v_{1}$ and $v_{2}$ belong to $\mathcal{S}$.

Defining a transposed diagram as the up-side-down reflected diagram, the scalar product $\left\langle s_{1} \mid s_{2}\right\rangle$ of two elements $s_{1}$ and $s_{2}$ of $\mathcal{S}$ can be defined as follows [8]. If one removes any loop from the diagram representing $s_{1}{ }^{T} s_{2}$, the resulting diagram is always $v_{1}$ with a factor depending on $s_{1}$ and $s_{2}$. The scalar product is given by this factor:

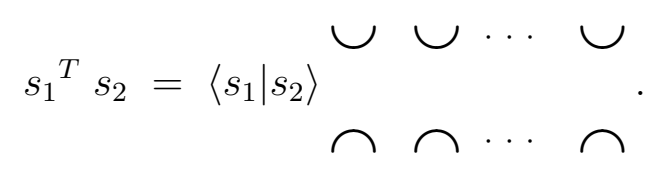

For example it is easy to see that

$$
\left\langle v_{1} \mid v_{1}\right\rangle=\left\langle v_{2} \mid v_{2}\right\rangle=x^{N / 2}
$$

Thus we can calculate the expectation value of the correlation operators $g_{l, n}^{ \pm}$for a state 
$v$ in $\mathcal{S}$. We evaluate the diagrams

$$
\begin{aligned}
v^{T} g_{l, m}^{ \pm} v=\left\langle v\left|g_{l, m}\right| v\right\rangle & \cup \cup \cdots \cup \\
& \frown \sim \cdots \curvearrowleft
\end{aligned}
$$

using the recurrence relation (2) expressed in terms of diagrams. This way by induction on $\mathrm{k}$, we can show that the correlation functions for the states $v_{1}$ and $v_{2}$ for all $N$ are given by

$$
\left\langle v_{i}\left|g_{l, l+k}^{ \pm}\right| v_{i}\right\rangle= \begin{cases}\left(x-\frac{1}{x}\right) x^{N / 2} & \text { for } i=1, k=1 \text { and } l \text { odd } \\ \left(x-\frac{1}{x}\right) x^{N / 2} & \text { for } i=2, k=1 \text { and } l \text { even } \\ & \text { and for } i=2, l=1, l+k=N \\ 0 & \text { otherwise. }\end{cases}
$$

Thus only sites connected by an upper line in $v_{1 / 2}$ have a non-zero correlation.

Note that we find trivial short range correlations, although the operators $g_{l, l+k}^{ \pm}$have a non-local structure (2). The ground states do not have any long range order. This can be expected from the interpretation of the words $v_{1}$ and $v_{2}$ in terms of spins that we give in the following paragraph for the representation (16) with spin $\frac{1}{2}$.

So far we have used the regular representation of the Temperley-Lieb algebra producing results independent of special representations. We now take the $U_{q} S U(2)$ spin- $\frac{1}{2}$ representation of $T_{N}(q)$, that is defined by [8, 9]

$e_{l}=-\frac{1}{2}\left(\sigma_{l}^{x} \sigma_{l+1}^{x}+\sigma_{l}^{y} \sigma_{l+1}^{y}+\frac{q+q^{-1}}{2}\left(\sigma_{l}^{z} \sigma_{l+1}^{z}-1\right)+\frac{q-q^{-1}}{2}\left(\sigma_{l}^{z}-\sigma_{l+1}^{z}\right)\right)$

where $\sigma^{x}, \sigma^{y}$ and $\sigma^{z}$ are Pauli matrices. For this representation the $g_{l, m}^{ \pm}(2)$ are $U_{q} S U(2)$ invariant generalizations of the $S U(2)$ invariant scalar product [8]

$$
g_{l, m}^{q=1}=-\frac{1}{2} \vec{\sigma}_{l} \cdot \vec{\sigma}_{m}
$$

The resulting Hamiltonian $H^{(N)}$ is $U_{q} S U(2)$ invariant. One would like to know the spin configuration states that correspond to the boundary diagrams $v_{i}$, i.e. the ground states of this quantum chain for conditions (8) and (11). To this end consider the special case

$$
b(q)=0 \text { and } a(q)=2 /\left(x^{2}-2\right) .
$$

With the chosen representation and this condition $H^{(N)}$ becomes the Hamiltonian of the $q$-deformed Majumdar-Ghosh model [2, 6] given by

$$
H^{\mathrm{MG}}=\sum_{i=1}^{N-2} P_{i, i+1, i+2}^{3 / 2}
$$


where $P_{i, i+1, i+2}^{3 / 2}$ is the projector onto the ( $q$-deformed) quartet of the spins at site $i, i+1$ and $i+2$.

The Majumdar-Ghosh model is known to have a valence-bond ground state [3, 4, 6]. Denoting the spin- $\frac{1}{2}$ representation space of a site by a dot and a singlet combination of two adjacent spins by a short line, they can be given pictorially as

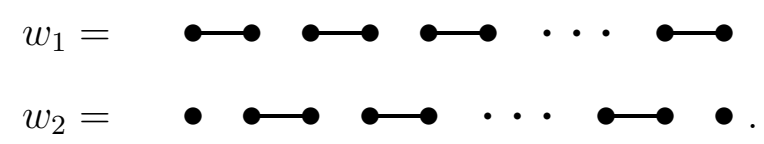

for an even number of sites. For $N$ odd the ground state has the form of (21) without the dot to the very left or very right.

One can easily verify that $w_{1}$ and $w_{2}$ are in fact ground states. The action of any $P_{i, i+1, i+2}^{3 / 2}$ on either of these states gives zero, since two of the three spins of sites $i, i+1$ and $i+2$ are in a singlet configuration. Thus both states are eigenvectors of $H^{\mathrm{MG}}$ with zero eigenvalue. Also all possible eigenvalues of $H^{\mathrm{MG}}$ are greater or equal to zero, because it is a sum of projectors.

On the other hand we have the ground states $v_{1}, v_{2}$ and $v_{3}$ (respectively $v_{4}$ or $v_{5}$ for $N$ odd), where $v_{1}$ and $v_{2}$ belong to the ideal $\mathcal{S}$. For the representation (16) $\mathcal{S}$ is known to represent the $U_{q} S U(2)$ scalar states $[7,8]$.

At this point the correspondence between the diagrams $v_{i}$ and spin configurations $w_{j}$ becomes clear. A line connecting two upper points in a boundary diagram is represented by the singlet configuration of the spins at the corresponding sites. For this identification one can easily show that the action of the Temperley-Lieb algebra on a diagram of $\mathcal{S}$ is mirrored by the action of matrices (16) on the matching spin state. In this way the two words $v_{1}$ and $v_{2}$ are represented by the two $U_{q} S U(2)$ ground state singlets $w_{1}$ respectively $w_{2}$ (with the spins of sites 1 and $N$ in the singlet combination). The correct normalization of the spin states can be calculated from (13). Further analyzing the action of $T_{N}(q)$ we find that the word $v_{3}$ corresponds to a linear combination of the ground state triplet and the singlet that are both pictorially described by $w_{2}$. Finally for $N$ odd, $v_{4}$ and $v_{5}$ correspond to $w_{2}$ without the left respectively right dot.

Recently generalizations of the Majumdar-Ghosh model have been discussed. A class of $S U(2)$ symmetric antiferromagnetic chains with valence-bond ground state can be found in [15]. Takano has introduced a generalization of the projection operators which appear in the formulation of the Majumdar-Ghosh model [16]. A q-deformation of the $S U(2)$ symmetric model has been given in [6]. With representation (16) the class of Hamiltonians $H^{(N)}$ is an extension of the latter $U_{q} S U(2)$ symmetric MajumdarGhosh chain. Note that the valence-bond ground states remain unchanged for a range of functions $a(q)$ and $b(q)$. A similar phenomenon was already found for a spin-1 chain with matrix product ground state [17]. We have not investigated, whether our Hamiltonian 
is massless or not [0]].

Choosing a different representation we can define further models with the same property. It is possible to represent a Temperley-Lieb algebra on quantum chains with $n=2 s+1$ states per site. Such a representation of $T_{N}(q)$ is given through the matrix elements [11

$$
\left\langle m_{i}, m_{i+1}\left|e_{i}\right| m_{i}^{\prime}, m_{i+1}^{\prime}\right\rangle=(-1)^{m_{i}-m_{i}^{\prime}} p^{m_{i}+m_{i}^{\prime}} \delta_{m_{i}+m_{i+1}, 0} \delta_{m_{i}^{\prime}+m_{i+1}^{\prime}, 0}
$$

where $m_{i}$ is the spin variable at site $i$ with $-s \leq m_{i} \leq s$. The value of $p$ can be calculated from

$$
q+q^{-1}=x=[n]_{p} \equiv\left(p^{n}-p^{-n}\right)\left(p-p^{-1}\right)^{-1} .
$$

For $s=1 / 2$ this reduces to the representation given in (16). The resulting Hamiltonians $H^{(N)}$ are $U_{p} S U(n)$ symmetric [11, 12]. In general the matrices (22) realize the projector onto the $U_{p} S U(n)$ singlet at two adjacent sites [10, 11] which is realized via the branching rule

$$
n \times \bar{n}=\left(n^{2}-1\right)+1 .
$$

Thus even for $s>1 / 2$ the ground state is of valence-bond type. For example we can expect the ground state of the $U_{p} S U(3)$ symmetric model to be tenfold degenerate (one octet and two singlets).

We have given the correspondence between specific words of the Temperley-Lieb algebra and vectors of the spin- $\frac{1}{2}$ configuration space. The general relation of words of $T_{N}(q)$ and spin states will be discussed in a future publication.

\section{Acknowledgments}

We would like to thank V. Rittenberg for suggesting the problem. Thanks also to M.T. Batchelor and F.H.L. Eßler for many helpful discussions.

\section{References}

[1] Temperley H N V and Lieb E H 1971 Proc. R. Soc. A 322251

[2] Majumdar C K and Ghosh D K 1969 J. Math. Phys. 101399

[3] Majumdar C K 1970 J. Phys. C: Solid State Phys. 3911

[4] van den Broek P M 1980 Phys. Lett. 77A 261

[5] Affleck I, Kennedy T, Lieb E H and Tasaki H 1988 Commun. Math. Phys 115477

[6] Batchelor M T and Yung C M $1994 q$-deformation of quantum spin chains with exact valence-bond ground state Preprint cond-mat 9403080

[7] Martin P P 1991 Potts Models and Related Problems in Statistical Mechanics (World Scientific, Singapore)

[8] Hinrichsen H, Martin P P, Rittenberg V and Scheunert M 1994 Nucl. Phys. B 415533 
[9] Pasquier V and Saleur H 1990 Nucl. Phys. B 330523

[10] Batchelor M T and Kuniba A 1991 J. Phys. A: Math. Gen. 242599

[11] Batchelor M T, Mezincescu L, Nepomechie R I and Rittenberg V 1990 J. Phys. A: Math. Gen. 23 L141

[12] Affleck I 1990 J. Phys.: Condens. Matter 2405

[13] Barber M N and Batchelor M T 1989 Phys. Rev. B 404621

[14] Anderson P W 1987 Science 2351196

[15] Shastry B S and Sutherland B 1981 Phys. Rev. Lett. 47964

[16] Takano K 1994 J. Phys. A: Math. Gen. 27 L269

[17] Klümper A, Schadschneider A and Zittartz J 1993 Europhysics Letters 24293 